\title{
Cancer Diagnosis, Treatment and Therapy
}

\section{Said Dermime*}

Biomedical Research Facility, Research Center, King Fahad Specialist Hospital Dammam, Kingdom of Saudi Arabia

Welcome to the June 2013 issue of the Journal of Carcinogenesis and Mutagenesis: Diagnosis, Treatment and Therapy of Cancer. This issue covers a number of comprehensive reviews focused on early detection and diagnosis of cancer, targeted therapy and treatment of cancer, chronotherapy, and cancer prevention in hard to cure malignancies. A better chance for curing cancer is through early diagnosis, detection and treatment. Apparent types of tumors such as melanoma and breast cancer usually can be identified before the progression of the disease by self-examination or other screening procedures. However, there are many cases of other types of cancers detected and diagnosed when disease progression and serious symptoms have already developed. A few cases of cancers are diagnosed while evaluating or treating other medical conditions.

Most routine screening procedures for cancer detection are mainly based on examination of cell morphology, tissue histology and measurement of body fluids markers, which lack sufficient sensitivity and/or specificity for early detection of cancer. Indeed, most secreted proteins studied as cancer screening biomarkers have low sensitivity and/or low specificity and this could be due to the use of non-sensitive tools or due to the fact that many of these tumor markers are also produced by non-cancerous cells or tissues. Altogether, these facts support the urgent need for the discovery of innovative tools and novel tumor markers for cancer screening, diagnosis and targeted therapy. Recently, scientists and clinicians have shifted to innovative techniques in order to identify and characterize biomarkers that drive the development and progression of cancer, and to discover upstream genes/proteins which could be useful to detect early-stage cancer, predict prognosis, determine therapy efficacy, or to be novel drug targets.

This special issue of the journal focuses on the diagnosis, treatment and therapy of cancer in which four different but complimentary areas are addressed.

The first subject addresses the early detection and diagnosis of cancer. In this, Ghebeh and Al-Alwan [1] discussed the role of Cancer Stem Cells (CSCs) in the generation of immune suppressive microenvironment that can lead to immune escape of cancer. It is now accepted that cancer originates and sustains by CSCs which share many features of normal stem cells including self-renewing ability and the immune privilege properties of normal stem cells. Possession of these features by CSCs contributes to failure of treatment and tumor relapse. Ghebeh and Al-Alwan [1] also highlight the recent advances in understanding the mechanisms which regulate the immunomodulatory properties of CSCs in order to develop more effective therapy that can eradicate these cells. Khadir and Tiss [2] on the other hand discuss the most promising proteomics strategies and techniques that can shorten the way from bench to bedside in cancer proteomics. In this part, they address the recent proteomic technologies which hold immense promise in the search of new clinical biomarkers for the early detection and diagnosis of cancer in addition to their involvement in the discovery of new therapeutic targets. Khadir and Tiss, summarize in this part the various mass spectrometry-based proteomic approaches and techniques used in cancer studies, discuss the complexity and the critical steps in handling and processing biological samples, and also focus on future development of novel proteomic techniques that drive their application from bench to bedside and back.

The second subject focuses on targeted therapy and treatment of cancer. In this, Afreen and Al-Safran [3] address the use of biological (allogeneic stem cell transplantation) versus drugs (Tyrosine Kinase Inhibitors) for the treatment and therapy of leukemia. The authors discuss the treatment strategies for chronic myeloid leukemia (CML) that have been changed considerably after the introduction of tyrosine kinase inhibitors as a first line therapy in the last decades. Allogeneic stem cell transplantation remains the only cure at present even though a small fraction of patients has been reported to remain in molecular remissions after discontinuing of tyrosine kinase inhibitors. They also highlight the major limitations of transplantation and the areas of studies required to improve the clinical outcomes of CML patients. In the second part of this subject, Owens et al. [4] focus on the use of Inhibitor of Apoptosis Proteins (IAPs) which are commonly upregulated in cancer and have become the focal point of much research as a future potential therapeutic target for cancer treatment. These proteins act downstream of a broad range of stimuli, such as cytokines and extracellular matrix interactions, to regulate cell survival, proliferation and migration (these processes are dysregulated during tumourigenesis and are critical to the metastatic spread of the disease). They also discuss the roles that IAPs may play in cancer, and the potential benefits and drawbacks of targeting IAPs. Owens et al suggest that combining anti-IAPs therapies with traditional drug regimens should have remarkable promise for the future care of cancer patients.

In the third subject Boudjelal et al. [5] address the issue of designing a proper treatment protocol based on clock biology (chronotherapy). As many novel and next generation drugs target transcriptional regulation (nuclear receptors modulators, kinases inhibitors and epigenetic regulating enzymes) careful consideration should be given to the time and duration of activation of these pathways. Chronotherapy has been proven to be effective in cancer as it takes into consideration the timing of target expression, disease manifestation and the activation of the enzymatic machinery involved in drug metabolism. The authors also specify the function of the molecular clock in health and disease with special reference to cancer and describe how chronotherapy can be applied in the clinical setting to treat cancer patients.

In the fourth subject $\mathrm{Al}$ Saghier et al. [6] address the issue of cancer prevention -prevention is better than cure. The general and common

*Corresponding author: Said Dermime, MSc., PhD. Senior Principal Scientist, Director of Biomedical Research Facility, Research Center, King Fahad Specialist Hospital Dammam, MBC-074, Bldg 100, Office 31, PO Box 15215, 31444 Dammam, Kingdom of Saudi Arabia, E-mail: said.dermime@kfsh.med.sa or sdermime@hotmail.com

Received May 27, 2013; Accepted May 28, 2013; Published June 10, 2013

Citation: Dermime S (2013) Cancer Diagnosis, Treatment and Therapy. J Carcinogene Mutagene S14: 007. doi:10.4172/2157-2518.S14-007

Copyright: (c) 2013 Dermime S. This is an open-access article distributed under the terms of the Creative Commons Attribution License, which permits unrestricted use, distribution, and reproduction in any medium, provided the original author and source are credited. 
cancer preventions include: avoiding/quitting smoking; avoiding too much sun exposure and wearing sunscreen outdoors; eating a nutritious/healthy diet; exercising regularly; getting proper cancer screening and taking proper precautions around carcinogens. In this part of this special issue the authors concentrated on gastric cancer which is the with one of the most common cause of cancer-related death in the world. Gastric cancer continue to be an important social and health problem worldwide, due to its high incidence, aggressiveness and low rate of cure. In addition, the disease has excessive poor prognosis and the main hope for cure at this time rests with surgical treatment. Therefore, attention should be given to preventive measures in addition to the development of novel treatment modalities in this malignancy. In this regards, priorities should be concentrated on the causatives of this type of cancer. Indeed, it has been demonstrated that several environmental risk factors play an important role throughout all the stages of the disease progression and treatment.

Finally, I would like to thank all authors who contributed to this special issue of the Journal of Carcinogenesis and Mutagenesis. I really hope that our readers such as clinicians, researchers and students will benefit from reading these reviews.

\section{References}

1. Ghebeh H, Al-Alwan M (2013) Do Cancer Stem Cells have an Immunomodulatory Role Different from the Bulk of Tumor Cells? J Carcinogene Mutagene S14 003. doi:10.4172/2157-2518.S14-003.

2. Khadir A, Tiss A (2013) Proteomics Approaches towards Early Detection and Diagnosis of Cancer. J Carcinogene Mutagene S14: 002. doi:10.4172/21572518.S14-002.

3. Afreen S, Safran ZA (2013) Allogeneic Stem Cell Transplantation for Chronic Myeloid Leukemia in the Era of Tyrosine Kinase Inhibitors- What are the Limitations? J Carcinogene Mutagene S14: 001. doi:10.4172/2157-2518.S14001.

4. Owens TW, Gilmore AP, Streuli CH, Foster FM (2013) Inhibitor of Apoptosis Proteins: Promising Targets for Cancer Therapy. J Carcinogene Mutagene S14: 004. doi:10.4172/2157-2518.S14-004

5. Boudjelal M, Mosskowsaka DE, Farrow S (2013) Interlink between Nuclear Receptors, Posttranslational Modifications and the Biological Clock in Health and Diseases. J Carcinogene Mutagene S14: 005. doi:10.4172/2157-2518. S14-005

6. Al Saghier A, Kabanja AH, Afreen S, Sagar M (2013) Gastric Cancer Environmental Risk Factors, Treatment And Prevention. J Carcinogene Mutagene S14: 005. doi:10.4172/2157-2518.S14-008 